\title{
1 Experience shapes social information use in foraging fish
}

2

3 Mike M. Webster* \& Kevin N. Laland

4

5 School of Biology, Harold Mitchell Building, University of St Andrews, St Andrews, Fife, U.K.

6 KY16 9TF

7

8 *Corresponding Author: Tel: 01344 461690; Email: mike.m.webster@gmail.com

9

10

11

12

13

14

15

16

17

18

19

20

21

22

23 SHORT TITLE: Experience and social information 
24 Many species of animal use social information, and in a variety of different contexts, but it is not

25 clear to what degree their ability to do this depends upon their prior experience of the association

26 between the behaviour of others and reward. We addressed this question in an experiment in

27 which two stickleback species (Gasterosteus aculeatus and Pungitius pungitius) were exposed to

28 a novel feeding task and then tested under a range of conditions. Using a fully-factorial training

29 design, fish were either fed from the surface or the bottom of their tank, and at the same time

30 were exposed to conspecifics feeding from the surface or bottom. At test, we showed that in

31 order to be able to use demonstrator behaviour to anticipate the presence of food at the surface,

32 test subjects needed first to have prior experience of both: sticklebacks responded to the

33 behaviour of conspecifics that were feeding at the surface by rising higher in the water column

34 themselves, but, crucially, they only did this if they had prior experience both of finding food at

35 the water surface and of seeing others feed there. Moreover, they only displayed this response in

36 the presence of feeding conspecifics, but not when the demonstrators were not feeding or were

37 absent. The role of prior experience and learning in social information use is surprisingly

38 understudied. We suggest that such work is vital if we are to understand the level at which

39 natural selection operates in shaping social information use and social learning.

40

41 KEY WORDS: Producer-Scrounger; Public information; Social information; Social learning;

42 Social transmission;

43 
46 Animals can acquire information about the distribution of resources and hazards in their

47 environment via the behaviour of others. Social information use is well documented in a range of

48 species, with animals paying attention and responding to information from both con- and

49 heterospecifics (Galef \& Giraldeau 2001; Valone \& Templeton 2002; Danchin et al. 2004; Dall

50 et al. 2005; Valone 2007; Rendell et al. 2013; Hoppitt \& Laland 2013; Ward \& Webster 2016;

51 Webster \& Laland 2017). An important question for researchers interested in the transmission of

52 social information is: how does an individual's past experience shape its ability to recognise and

53 respond appropriately to social cues that convey relevant information?

54

55 Many species form groups for a variety of different reasons (Krause \& Ruxton 2002; Webster \&

56 Ward 2016), and social attraction alone may lead individuals to encounter and learn about the

57 presence or quality of resources the resources that others are using (Atton et al. 2012). Over time,

58 individuals that have often previously found resources when joining others may become even

59 more likely to join others. In house sparrows (Passer domesticus), birds that had previously

60 found food when foraging alongside others (taxidermy models in these experiments), were more

61 likely to join others when foraging subsequently (Katsnelson et al. 2008; Belmaker et al. 2012).

62 Bumblebees (Bombus terrestris) can acquire flower colour preferences after joining conspecifics

63 and subsequently finding food on novel flowers of a given colour (Dawson 2013; Avarguès-

64 Weber \& Chittka 2014). Through foraging near others, animals may come to form associations

65 between the presence of others and the distribution of resources. Beyond simple social attraction,

66 animals might use further cues when deciding when and who to join. They may be more strongly

67 attracted to larger or denser groups (Frommen et al. 2009), with the distribution of such

68 aggregations in turn being shaped the distribution of resources in the environment. There is 
69 increasing evidence that social attraction is plastic, and can be affected by social experience both

70 early on in development as juveniles (Chapman et al. 2008; Boogert et al. 2014) and in adulthood

71 (Swaney et al. 2001). Animals may also be more attracted to groups containing active compared

72 to inactive individuals, or those containing individuals exhibiting cues such as postures or

73 movements associated with feeding or competing (Coolen et al. 2001), behavioural biases that

74 may well be affected by experience.

75

76 In this study we explored the importance of both exposure to conspecific feeding-specific

77 behaviour and personal experience of finding food in the tendency of fish (three- and nine-spined

78 stickleback fish Gasterosteus aculeatus and Pungitius pungitius) to subsequently respond to

79 social cues indicating the presence of food at the surface of the water. We have used these

80 species as a model organisms for investigating social information use and learning for a number

81 of years, finding that both are capable social information users, but also that they differ in their

82 ability to learn from social cues (Laland et al. 2011). Both species are generalist foragers,

83 capturing prey from the substrate and water column and feeding from the surfaces of rocks,

84 plants and other structures (Bell \& Foster 1994). While neither species generally feeds from the

85 surface of the water, we show here that both can be trained to do so, in this case from the

86 underside of a floating tile.

87

88 In the experiment described in this paper we controlled the exposure of test subjects to both the

89 presence of food at the surface and the social cues provided by others feeding there, such that at

90 the end of the exposure period, each fish had been exposed to one of four conditions: 1 . The test

91 subject had experience of both feeding at the surface and of seeing conspecifics do so too. 2. It 
92 had experience of feeding from the surface, but had only ever seen conspecifics feed from the

93 substrate material at the bottom of its housing tank. 3. It had no experience of feeding from the

94 surface but had seen conspecifics do so. 4. It had neither fed from the surface, nor seen

95 conspecifics do so. We tested the hypothesis that the ability to use social information about the

96 presence of food at the surface would depend upon test subjects having experience of both

97 finding food there themselves and of previously seeing others feed there. We predicted that when

98 tested subjects would rise higher in the water column only if they had this joint experience

99 (condition 1), and only if demonstrators were present and feeding at the surface too.

101 METHODS

102

103 Subjects and housing

104

105 Several hundred three- and nine-spined sticklebacks were collected from Melton Brook in

106 Leicester, UK in October 2008. They were captured using dip nets and transported to our

107 laboratory at the University of St Andrews. Fish were held in single species groups of 50 fish in

$10890 \mathrm{~L}$ aquaria. Each aquarium contained a layer of gravel, artificial plants and was equipped with

109 an internal filter. Water temperature was held at $8^{\circ} \mathrm{C}$, and the light regime was $12: 12$, with the

110 room illuminated between $7 \mathrm{AM}$ and $7 \mathrm{PM}$. The fish were fed daily to satiation with frozen

111 bloodworms and Artemia. They were held under these conditions for several weeks until the

112 experiment began. All fish used in the experiment described below were adults measuring 35-40

$113 \mathrm{~mm}$ in length. We did not use fish displaying signs of being in reproductive condition, since this 
114 has been shown to affect social information use in other contexts (Webster \& Laland 2011). The

115 experiments described below took place between November 2008 and May 2009.

117 Design overview

119 Fish were trained and tested in aquaria that were divided into two sections, one containing the 120 test subject and the other the demonstrators (Figure 1). In the training phase of the experiment 121 individual test subjects were either presented with food at the surface of their half of the tank or

122 on the floor, and were also given the opportunity to watch conspecific demonstrators feed from

123 the surface or floor in the other half of the tank, as specified by conditions 1-4 described below.

124 In the testing phase they were exposed either to conspecifics that were feeding at the surface, 125 conspecifics that were not feeding, or they were tested alone. To ensure that subjects' behaviour 126 at test was not biased by the presence of food, no food was present in the test subject's half of the 127 test tank. We used the height of the fish above the substrate as the response variable. Both 128 species tend to remain close to the substrate when not feeding. We therefore expected fish to 129 only rise close to the surface when feeding or when expecting food, making this a reliable 130 indicator of food anticipatory behaviour.

132 Training tank and procedure

134 Individual test subjects were trained, or otherwise exposed to the floating tile and demonstrators, 135 in cube-shaped aquaria measuring $30 \mathrm{~cm}$ along each axis. Each aquarium was divided into two 136 sections using a clear plastic tank divider (Penn Plax brand), with five 2mm-diameter holes per 
137 square $\mathrm{cm}$. A 1-cm-deep layer of sand was added to each aquarium and each was filled with

138 water to a depth of $27 \mathrm{~cm}$. An air stone was added to each side of the aquarium to aerate the

139 water. On the surface of each of the two halves of the aquarium a $10 \mathrm{~cm}$-square sheet of white

140 polystyrene was floated. Each aquarium was completely surrounded with black paper. This

141 prevented the test subjects from receiving cues from other fish in adjoining aquaria. The test

142 subject was added to one half of the aquarium, while a group of three conspecific demonstrators

143 were added to the other half. The test subject and demonstrators were held in these tanks for 2

144 weeks until the test subjects were used in the experiment. They were fed twice per day, five

145 times per week, from Monday until Friday. Subjects were not fed at the weekends. The provision

146 of food to the test subjects and demonstrators varied between the four experimental treatments as

147 follows (see also Figure 1):

148

149 1. Test subjects and demonstrators both fed from tile. The polystyrene tiles were removed

150 from the aquaria and dried using paper towels. To each tile a small amount of Vaseline was

151 applied. This was used to stick thawed bloodworms to the underside of the tiles, 5 bloodworms

152 for test subject and 15 for demonstrators. The tiles were then placed back in the aquaria,

153 bloodworms facing down, and the fish were allowed to feed from them. After one hour the tiles

154 were removed and replaced with clean ones, containing no Vaseline or food. Fish were initially

155 reluctant to feed from the underside of the tiles during the first few days of the training, though

156 most fish did feed within the hour. Within a week or so all of the fish readily fed from the tiles

157 consuming most of the food within the first few minutes.

158 
159 2. Test subjects fed from tile, demonstrators fed from substrate. The polystyrene tiles were

160 removed, and Vaseline was applied to them as described above, but bloodworms were only

161 added to the tile of the test subject. When the tiles were returned to the aquaria, the

162 demonstrators were provided with 15 bloodworms on the surface of the sand substrate. These

163 were applied using a large pipette, with the food contained within $2 \mathrm{~cm}^{3}$ of tank water.

3. Test subjects fed from substrate, demonstrators fed from tile. Feeding was performed as in

166 treatment 2, above, except here it was the test subject that received five bloodworms delivered to

167 the sand substrate, while the demonstrators food was provided on the underside of the tile.

4. Test subjects and demonstrators both from substrate. The tiles were removed and Vaseline

170 applied to them as above, but both the test subjects and the demonstrators received their food via

171 pipette to the sand substrate, five and 15 bloodworms, respectively.

172

173 In total, 360 test subjects (180 of each species) were trained, 45 in each of these training

174 conditions. These in turn were tested in the 3 experimental treatments described below. 30

175 training tanks were established, and arranged into 6 blocks of 5 tanks each. Within each block 5

176 fish of the same species received the same training (in one of the four training conditions

177 described below) and were then tested in one of three test conditions described below. This was

178 repeated over 12 cycles until 360 fish had been trained and tested. The training and testing

179 schedule is presented in Table A1.

180

181 Test tank and procedure 
183 The test tank was similar to the training tank, with the exception that this time a $12 \mathrm{~cm}-\mathrm{base}, 30$ -

$184 \mathrm{~cm}$-tall transparent Perspex container was present in the demonstrator half of the aquarium. This

185 was watertight and was used to prevent the test subject from being able to detect chemical cues

186 from the bloodworms that were present in two of the treatments described below. The top $2 \mathrm{~cm}$

187 of the container was covered with black PVC tape. This prevented the test subject from being

188 able to see any bloodworms that were attached to the underside of the demonstrators' tile, but

189 still allowed them to see the demonstrators feeding. Three sides of the test tank were covered

190 with black paper to prevent outside disturbance. One side was left uncovered. This allowed us to

191 film through the side of the tank using a digital video camera. The test tank was filled with water

192 to a depth of $27 \mathrm{~cm}$. Horizontal lines $1 \mathrm{~cm}$ apart were drawn on the side of the tank facing the

193 camera. These allowed us to record the height in the water column of the test subject, our

194 response variable, as described below. In cases where the test subject was level with one of the

195 lines we used the height of the fish's eye relative to the line as a guide- if the eye was above or

196 below the line then the fish was recorded as above or below. If the eye was level with the line the

197 fish was always recorded as below. In all treatments a tile with Vaseline on it was present in the

198 test subject's half of the tank, but this never contained food. We performed 3 experimental

199 treatments, testing 15 fish per species from each of the four training conditions:

200

201 1. Demonstrators present and feeding. Three conspecific demonstrators were present and

202 allowed to feed from 15 blood worms stuck with Vaseline to the underside of the polystyrene

203 tile. 
205 2. Demonstrators present but not feeding. Three demonstrators were again present, but this

206 time no food was provided on the tile.

208 3. No demonstrators present. No demonstrators were present but food was present on the tile.

209 Though the design of the experiment prevented the fish from being able to see or smell the food,

210 including food in the no demonstrators conditioned allowed us to rule out the use of other cues,

211 such as discolouration of the water that may have been caused by the food.

212

213 Trials proceeded as follows. First the test subject was added to one half of the tank, and the

214 demonstrators (where present) were added to the container in the centre of the other half. These

215 were allowed to settle for 15 minutes. A polystyrene tile was then added to each half of the tank,

216 in the case of the demonstrators at the surface of the container. This contained a small amount of

217 Vasoline spread on the downward facing side. 15 blood worms were stuck to the tile in the

218 demonstrator container in one treatment, as described above, otherwise no food was present.

219 Adding the tiles did not seem to startle the fish. This marked the beginning of the experiment,

220 which lasted for a further five minutes. Each trial was recorded and from the videos we measured

221 the height of the test subject above the substrate to the nearest $\mathrm{cm}$ every minute, giving a total of

2225 measurements per trial.

223

224 Statistical analysis

225

226 We saw no trends for increasing or decreasing height of the test subject above the bottom of the

227 experimental arena during the observation period (Figures A1 \& A2). We therefore used the 
228 mean height of the test subject as the response variable in our analysis. Data were analysed using

229 a GLM. Test subject experience, demonstrator cue treatment and species were included as fixed

230 factors, with interactions between all factors also included. Tukey HSD post-hoc tests were used

231 to identify differences between test-subject experience and demonstrator-cue treatments.

232

233 Ethical Note

234

235 The study adhered to ASAB/ABS guidelines for the use of animals in research. This project was 236 approved by the University's Animal Welfare and Ethics Committee. No procedures required

237 U.K. Home Office licensing were performed. No animals exhibited signs of stress or illness

238 during these experiments. At the end of this project the fish were retained in the laboratory for 239 use in other work.

240

241 RESULTS

242

243 A GLM revealed main effects of demonstrator-cue treatment and test-subject experience, and an

244 interaction between these. We saw no difference between the two species, but there was an

245 interaction between species and demonstrator-cue treatment. There was no interaction between

246 species and test-subject experience, nor any three way interaction between these variables (Table

$2471 \&$ Figure 2). Among the demonstrator-cue treatments, fish swam higher in the water column in 248 the treatment where demonstrators were present and feeding compared to treatments where

249 demonstrators were present but not feeding or when they were absent. We saw no difference

250 between the latter two treatments (Table 2). In terms of test-subject experience, fish that had 
251 previously both fed from a floating tile and seen conspecifics feed from one swam higher than

252 fish in the other three conditions. We saw no pairwise differences between conditions 2 and 3 ,

253 conditions 2 and 4 , or conditions 3 and 4 (Table 3). These effects explain the interaction

254 observed in the analysis (Figure 2): across both species, fish only rose high in the water column

255 when they could see others feeding and when they had prior experience of both feeding from a

256 floating tile and seeing conspecifics do the same.

257

258 DISCUSSION

259

260 This experiment addressed the question of how observer experience of both reward and

261 demonstrator behaviour shapes subsequent social information use. We showed that in order to be

262 able to use demonstrator behaviour to anticipate the presence of food at the surface, the observers

263 needed first to have prior experience of both: sticklebacks that could not see or smell food

264 responded to the behaviour of conspecifics that were feeding close to the surface of the water by

265 rising higher in the water column themselves, but, crucially, they only did this if they themselves

266 had earlier both found food at the water surface and seen others feed there. This suggests that the

267 fish have learned that the presence or some aspect of the behaviour of demonstrators in the upper

268 levels of the water column predicted the arrival of food at the surface. Experienced individuals

269 only displayed this response in the presence of feeding conspecifics, but not when the

270 demonstrators were not feeding (and were not close to the water surface), or when demonstrators

271 were absent. Fish from the other observer experience treatments, those that lacked the double

272 experience of both feeding at the water surface and seeing others feeding there exhibited no

273 tendency to rise higher in the water column when tested, even when feeding demonstrators were 
274 present. This is consistent with associative learning, with the fish having learned an association

275 between the floating feeder and a food reward and demonstrator behaviour and the feeder,

276 leading to an association between demonstrator behaviour and food. Perhaps more simply, the

277 fish may have learned that demonstrators moving towards the surface predict the arrival of food

278 there. Further work is needed to disentangle these effects.

279

280 We saw no differences in the behaviour of the two species, though we did see an interaction

281 between species and treatment. The nature and biological significance of this interaction is

282 somewhat unclear however, with the nine-spined sticklebacks that had not been trained to feed

283 from the tile tending to remain at a lower level above the substrate than did similarly trained

284 three-spined sticklebacks. This may simply reflect fine-scale differences in the habitat

285 preferences of the two species; nine-spined sticklebacks have previously been shown to prefer

286 structured over open environments (Coolen et al. 2003; Hart 2003; Webster et al. 2009) and in

287 the experimental setting in the absence of other cover, they might have remained closer to the

288 substrate as a form of cover-seeking behaviour. In terms of social information use and

289 experience however, the responses of the two species did not appear to differ. This contrasts with

290 earlier work examining social information use and social learning in other contexts in these

291 species (Coolen et al. 2003), in which nine-spined sticklebacks, but not three-spined sticklebacks

292 were shown to able to use public information transmitted through demonstrator foraging

293 behaviour in order to select the richer of two prey patches. 
295 The role of prior learning of associations in the shaping of subsequent social information use has

296 arguably been understudied compared to other aspects of social information use and learning,

297 and warrants further investigation (Reader 2016; Leadbeater 2015; Leadbeater \& Dawson 2017).

298 An elegant example of one experiment that has directly investigated the processes behind social

299 learning concerns flower preference learning in bumblebees (Bombus terrestris). Dawson et al.

300 (2013) showed that social learning of flower colour could be explained by classical conditioning

301 and the integration of two learned associations, first the presence of conspecific on a flower of a

302 particular colour and second joining a conspecific and receiving a food reward. This experiment

303 demonstrates that social learning in bumblebees can be explained without the need to invoke

304 adaptively specialised social learning mechanisms. This is an interesting finding since recent

305 debates around social learning and its underlying cognitive mechanisms have questioned the

306 extent to which the distinction between social and non-social learning is meaningful or useful.

307 Some researchers have discussed whether, in many cases, social learning might operate through

308 domain-general (i.e. not adaptively-specialised) psychological mechanisms, with social learning

309 being an exaptation, i.e. an adaptive manifestation of pre-existing adaptations for learning. If this

310 is so then social learning is social only in a functional sense, in so far as information is

311 channelled through a social source (Lefebvre \& Giraldeau 1996; Leadbeater 2014; Heyes \&

312 Pearce 2015; Reader 2016). Work such as Dawson et al.'s (2013) bumblebee experiments,

313 Katsnelson et al.'s (2008) and Belmaker et al.'s (2012) producer-scrounger experiments with

314 sparrows, and our current study demonstrate that past experience plays an important role is

315 shaping subsequent social information use. They are consistent with a domain-general,

316 associative basis for these forms of social learning. However, given the relative paucity of

317 empirical work specifically addressing this question, further carefully-designed experiments in 
318 other species, and exploring social learning in contexts other than foraging are needed in order to

319 determine how broadly such findings apply.

321 Related work should also investigate biases in the so-called input channels (i.e. perceptual

322 processes) that determine how effectively animals detect, pay attention to, and respond to, social

323 information, and whether, in some species, these may be biased towards social sources (Heyes

324 2012). A basic question and useful starting point for such work concerns whether learning occurs

325 more rapidly or with greater accuracy when information is transmitted via a social versus non-

326 social channels. In our study test subjects with the right combination of experience readily

327 responded to feeding conspecifics by displaying food anticipatory behaviour themselves, but it is

328 not clear whether they would have formed this association as quickly, or even at all, had they

329 been exposed to a similarly-behaving non-biological stimulus, instead. In principle the fish could

330 be exposed to the simultaneous presence of both an artificial or abstract stimulus and a food

331 reward and tested using a similar protocol to the one deployed in the current study. Similar

332 approaches have been used to study social learning of artificial flower type preferences in

333 bumblebees. Here, Avarguès-Weber \& Chittka (2014) found that bumblebees that had previously

334 observed and joined other bumblebees as they fed from artificial flowers learned preferences for

335 artificial flowers of the same colour and visited these both when other bumblebees occupied

336 them and also when the flowers were unoccupied. When the test subjects had been allowed to

337 watch and then visit flowers attended by model bumblebees or bumblebee-sized white blocks

338 however they learned to 'join' these stimuli when they were present on the flowers but they

339 failed to acquire a preference for unoccupied flowers of the demonstrated colour as they did

340 when exposed to live demonstrators. Smolla et al. (2016) report that when resource distribution 
341 was unstable bumblebees copied the flower choices of realistic model bumblebees but that they

342 did not copy the flower choices of an unnatural object, a green rubber cuboid. More research in

343 this area would be useful and such work could provide useful information on whether animals in

344 general learn more readily from social sources or channels, and if so, how and why. Input

345 channel biases may plausibly arise through adaptive specialisation, with natural selection

346 favouring the ability to detect, filter and attend to pertinent cues in the behaviour of others, as for

347 example appears manifest in the particular sensitivity of rhesus macaques (Macaca mulatta) to a

348 snake stimulus in observational conditioning of fears (Mineka \& Cook, 1988). However, such

349 biases could also plausibly arise through individual experience as well, and research that

350 attempts to separate and account for the relative contribution of adaptive specialisation and

351 learning would be valuable.

352

353 In our study we exposed our wild-captured test subjects to an artificial challenge, one we could

354 be reasonably certain they had not encountered before, structuring their opportunity to engage

355 with the task, and to observe others interacting with it, in a controlled manner. It is worth noting

356 that in most studies of social learning, whether conducted on wild-captured or captive-bred

357 animals, the researchers lack detailed data on the previous experience of their test subjects,

358 experience that may well shape the behaviour exhibited at test. To overcome this potential

359 problem, researchers might in principle use animals that have been bred and raised in a

360 controlled and constantly monitored environment, allowing researchers to compile a complete

361 record of their interactions with their physical and social environment and to quantify their

362 history of exposure to social cues. By studying replicated populations from birth to adulthood

363 under as close as possible to natural conditions, and by collecting physiological and behavioural 
364 data and performing appropriate controls, researchers should be able account for the relative

365 importance of development, opportunity to learn privately and from others, and of adaptive

366 specialisation in shaping social information use and learning. While in practice such a project

367 would be a major undertaking, the means for long-term tracking and collating of such data are

368 now well within the reach of researchers (e.g. Dell et al. 2014, Hong et al. 2015, Meikle \& Holst

369 2015, Peters et al. 2016, Gernat et al. 2018), as are the statistical techniques for incorporating

370 such information into models that describe how and when individuals acquire novel information

371 and learn new behaviour patterns (Hoppitt \& Laland 2013). Ultimately, such intensive

372 experiments may be necessary if we are to fully grasp the proximate and evolutionary bases of

373 social learning.

374

375

376

377 
379 Atton, N., Hoppitt, W., Webster, M.M., Galef, B.G. \& Laland, K.N. (2012). Information flow 380 through threespine stickleback networks without social transmission. Proceedings of the Royal 381 Society of London B: Biological Sciences, rspb20121462.

382

383 Avarguès-Weber, A. \& Chittka, L. (2014). Local enhancement or stimulus enhancement?

384 Bumblebee social learning results in a specific pattern of flower preference. Animal behaviour, $38597,185-191$.

386

387 Bell, M.A. \& Foster, S.A. (1994). The Evolutionary Biology of the Threespine Stickleback.

388 Oxford: Oxford University Press.

389

390 Belmaker, A., Motro, U., Feldman, M.W. \& Lotem, A. (2012). Learning to choose among social 391 foraging strategies in adult house sparrows (Passer domesticus). Ethology, 118(11), 1111-1121. 392

393 Boogert, N.J., Farine, D.R. \& Spencer, K.A. (2014). Developmental stress predicts social 394 network position. Biology letters, 10, 20140561.

395

396 Chapman, B.B., Ward, A.J.W. \& Krause, J. (2008). Schooling and learning: early social 397 environment predicts social learning ability in the guppy, Poecilia reticulata. Animal Behaviour, 398 76, 923-929. 
400 Coolen, I., Giraldeau, L.A. \& Lavoie, M. (2001). Head position as an indicator of producer and 401 scrounger tactics in a ground-feeding bird. Animal Behaviour, 61, 895-903.

402

403 Coolen, I., Bergen, Y.V., Day, R.L. \& Laland, K.N. (2003). Species difference in adaptive use of 404 public information in sticklebacks. Proceedings of the Royal Society of London B: Biological 405 Sciences, 270, 413-2419.

406

407 Danchin, E., Giraldeau, L.A., Valone, T.J. \& Wagner, R.H. (2004). Public information: from 408 nosy neighbors to cultural evolution. Science, 305, 487-491.

409

410 Dall, S.R., Giraldeau, L.A., Olsson, O., McNamara, J.M. \& Stephens, D.W. (2005). Information 411 and its use by animals in evolutionary ecology. Trends in Ecology and Evolution, 20, 187-193.

413 Dawson, E.H., Avarguès-Weber, A., Chittka, L. \& Leadbeater, E. (2013). Learning by

414 observation emerges from simple associations in an insect model. Current Biology, 23, 727-730.

415

416 Dell, A.I., Bender, J.A., Branson, K., Couzin, I.D., de Polavieja, G.G., Noldus, L.P., Pérez-

417 Escudero, A., Perona, P., Straw, A.D., Wikelski, M. \& Brose, U. (2014). Automated image418 based tracking and its application in ecology. Trends in Ecology and Evolution, 29, 417-428.

420 Frommen, J.G., Hiermes, M. \& Bakker, T.C. (2009). Disentangling the effects of group size and 421 density on shoaling decisions of three-spined sticklebacks (Gasterosteus aculeatus). Behavioral 422 Ecology and Sociobiology, 63, 1141-1148. 
424 Galef, B.G. \& Giraldeau, L.A. (2001). Social influences on foraging in vertebrates: causal

425 mechanisms and adaptive functions. Animal Behaviour, 61, 3-15.

426

427 Gernat, T., Rao, V.D., Middendorf, M., Dankowicz, H., Goldenfeld, N. \& Robinson, G.E.

428 (2018). Automated monitoring of behavior reveals bursty interaction patterns and rapid

429 spreading dynamics in honeybee social networks. Proceedings of the National Academy of

$430 \quad$ Sciences, 115, 1433-1438.

431

432 Hart, P.J.B (2003). Habitat use and feeding behaviour in two closely related fish species, the

433 three-spined and nine-spined stickleback: an experimental analysis. Journal of Animal Ecology, $43472,777-783$.

435

436 Heyes, C. (2012). What's social about social learning? Journal of Comparative Psychology, 126, $437 \quad 193-202$.

438

439 Heyes, C. \& Pearce, J.M. (2015). Not-so-social learning strategies. Proceedings of the Royal

440 Society of London B: Biological Sciences, 282, 20141709.

441

442 Hong, W., Kennedy, A., Burgos-Artizzu, X.P., Zelikowsky, M., Navonne, S.G., Perona, P. \&

443 Anderson, D.J. (2015). Automated measurement of mouse social behaviors using depth sensing,

444 video tracking, and machine learning. Proceedings of the National Academy of Sciences, 112,

445 E5351-E5360. 
447 Hoppitt, W. \& Laland, K.N. (2013). Social learning: An Introduction to Mechanisms, Methods, 448 and Models. Princeton: Princeton University Press.

449

450 Katsnelson, E., Motro, U., Feldman, M.W. \& Lotem, A. (2008). Early experience affects

451 producer-scrounger foraging tendencies in the house sparrow. Animal Behaviour, 75, 1465-

4521472.

453

454 Kendal, R.L., Boogert, N.J., Rendell, L., Laland, K.N., Webster, M. \& Jones, P.L. (2018). Social 455 Learning Strategies: Bridge-Building between Fields. Trends in Cognitive Sciences, 22, 651-665. 456

457 Laland, K.N., Atton, N. \& Webster, M.M. (2011). From fish to fashion: experimental and 458 theoretical insights into the evolution of culture. Philosophical Transactions of the Royal Society 459 of London B: Biological Sciences, 366, 958-968.

460

461 Leadbeater, E. (2015). What evolves in the evolution of social learning? Journal of Zoology, $462 \quad 295,4-11$.

463

464 Leadbeater, E. \& Dawson, E.H. (2017). A social insect perspective on the evolution of social 465 learning mechanisms. Proceedings of the National Academy of Sciences, 114, 7838-7845. 
467 Lefebvre, L. \& Giraldeau, L.A. (1996). Is social learning an adaptive specialization? In C.M.

468 Heyes \& B.G. Galef (Eds.), Social Learning in Animals: the Roots of Culture (pp. 107-128).

469 London: Academic Press.

470

471 Meikle, W.G. \& Holst, N. (2015). Application of continuous monitoring of honeybee colonies.

472 Apidologie, 46, 10-22.

473

474 Mineka, S. \& Cook, M. (1988). Social learning and the acquisition of snake fear in monkeys. In

475 T. R. Zentall \& B.G. Galef (Eds.), Social learning: Psychological and Biological Perspectives

476 (pp.51-73). Hillsdale: Lawrence Erlbaum Associates.

477

478 Peters, S.M., Pinter, I.J., Pothuizen, H.H., de Heer, R.C., van der Harst, J.E. \& Spruijt, B.M.,

479 (2016). Novel approach to automatically classify rat social behavior using a video tracking

480 system. Journal of Neuroscience Methods, 268, 163-170.

481

482 Reader, S.M. (2016). Animal social learning: associations and adaptations. F1000 Research, 5.

483

484 Rendell, L., Fogarty, L., Hoppitt, W.J., Morgan, T.J., Webster, M.M. \& Laland, K.N. (2011).

485 Cognitive culture: theoretical and empirical insights into social learning strategies. Trends in

486 Cognitive Sciences, 15, 68-76.

487

488 Smolla, M., Alem, S., Chittka, L. \& Shultz, S. (2016). Copy-when-uncertain: bumblebees rely on

489 social information when rewards are highly variable. Biology Letters, 12, 20160188. 
491 Swaney, W., Kendal, J., Capon, H., Brown, C. \& Laland, K.N. (2001). Familiarity facilitates

492 social learning of foraging behaviour in the guppy. Animal Behaviour, 62, 591-598.

493

494 Valone, T.J. \& Templeton, J.J. (2002). Public information for the assessment of quality: a

495 widespread social phenomenon. Philosophical Transactions of the Royal Society Series B 357, $496 \quad 1549-1557$.

497

498 Valone, T.J. (2007). From eavesdropping on performance to copying the behavior of others: a 499 review of public information use. Behavioural Ecology and Sociobiology 62, 1-14.

500

501 Ward, A.J.W \& Webster, M.M. (2016). Sociality: The Behaviour of Group-Living Animals.

502 Zurich: Springer.

503

504 Webster, M.M. \& Laland, K.N. (2011). Reproductive state affects reliance on public information

505 in sticklebacks. Proceedings of the Royal Society of London B: Biological Sciences, 278, 619$506 \quad 627$.

507

508 Webster, M.M. \& Laland, K.N. (2017). Social information use and social learning in non509 grouping fishes. Behavioral Ecology, 28, 1547-1552.

510

511 Webster, M.M., Ward, A.J.W. \& Hart, P.J.B. (2009). Individual boldness affects interspecific 512 interactions in sticklebacks. Behavioural Ecology and Sociobiology, 63, 511-520. 


\section{TABLES}

514

515 Table 1. Factors affecting observer responses to social cues

$\begin{array}{lccc} & \boldsymbol{d} \boldsymbol{f} & \boldsymbol{F} & \boldsymbol{P} \\ & & & \\ \text { Treatment } & 2 & 65.40 & <0.001 \\ \text { Experience } & 3 & 112.64 & <0.001 \\ \text { Species } & 1 & 0.54 & 0.46 \\ \text { Treatment } * \text { Experience } & 6 & 49.83 & <0.001 \\ \text { Treatment * Species } & 2 & 3.07 & 0.05 \\ \text { Experience * Species } & 3 & 2.09 & 0.10 \\ \text { Treatment } * \text { Experience } * \text { Species } & 6 & 0.56 & 0.76 \\ \text { Total } & 360 & & \\ \text { Corrected } & 359 & & \\ \mathrm{R}^{2}=0.70 & & & \end{array}$

516

517 Table 1. Output from a GLM investigation the effects of demonstrator behaviour (Treatment),

518 test subjects' prior training (Experience) and species and the interactions between these upon test 519 subject water column position. See also Figure 2 and main text for further details.

520

521

522

523 
524 Table 2. Post-hoc comparison of demonstrator behaviour treatments

525

Comparison

P, F vs. P, NF

P, F vs. A

P, NF vs. A
$\boldsymbol{P}$

$<0.001$

$<0.001$

0.06
95\% CI lower, upper bounds

$1.58,2.77$

$2.15,3.34$

$-0.03,1.17$

526

527 Table 2. Tukey HSD post-hoc tests comparing the effects of the three different demonstrator

528 behaviour treatments (P, F: conspecifics present and feeding; P, NF: present but not feeding; A:

529 conspecifics absent). See also Figure 2 and main text for further details.

530

531

532

533

534

535

536

537

538

539

540

541 
542 Table 3. Post-hoc comparison of observer treatments

543

Comparison

1 vs. 2

1 vs. 3

1 vs. 4

2 vs. 3

2 vs. 4

3 vs. 4
$P$

$<0.001$

$<0.001$

$<0.001$

0.31

0.78

0.87
95\% CI lower, upper bounds

$3.37,4.89$

$3.87,5.40$

$3.65,5.17$

$-0.25,1.26$

$-0.48,1.03$

$-0.98,0.53$

544

545 Table 3. Tukey HSD post-hoc tests comparing the effects of the four different observer

546 behaviour treatments (1. Experience of feeding from floating tile and seeing others eat from it; 2.

547 Experience of feeding from floating tile but not of seeing others eat from it; 3 . No experience of

548 feeding from tile, but has seen others feed from it; 4 . No experience of feeding from time or

549 seeing others feed from it). See also Figure 2 and main text for further details.

550

551

552

553

554

555

556 
557 Table A1. Testing schedule

558

Date Treatment

Block A Block B Block C Block D Block E Block F

Nov/2008 $\quad 9 s s / 1 / 2 \quad 9 s s / 2 / 2 \quad 3 s s / 3 / 2 \quad 3 s s / 1 / 2 \quad 9 s s / 2 / 1 \quad 3 s s / 3 / 1$

$\begin{array}{lllllll}\text { Nov/2008 } & 3 \mathrm{ss} / 1 / 1 & 9 \mathrm{ss} / 4 / 1 & 9 \mathrm{ss} / 4 / 3 & 9 \mathrm{ss} / 3 / 1 & 9 \mathrm{ss} / 1 / 1 & 9 \mathrm{ss} / 2 / 1\end{array}$

Dec/2008 3ss/4/2 $\quad 9 s s / 3 / 2 \quad 3 s s / 1 / 1 \quad 3 s s / 2 / 2 \quad 3 s s / 4 / 3 \quad 3 s s / 3 / 3$

$\operatorname{Jan} / 2009 \quad 3 s s / 3 / 2 \quad 9 s s / 1 / 2 \quad 3 s s / 2 / 1 \quad 3 s s / 2 / 2 \quad 3 s s / 3 / 1 \quad 3 s s / 1 / 3$

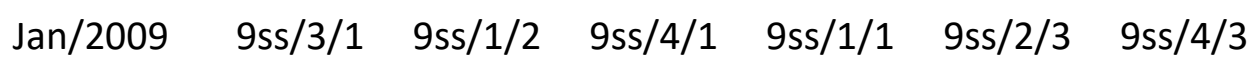

Feb/2009 $\quad 9 s s / 3 / 1 \quad 9 s s / 1 / 3 \quad 3 s s / 1 / 3 \quad 9 s s / 4 / 2 \quad 3 s s / 4 / 1 \quad 3 s s / 2 / 3$

$\begin{array}{lllllll}\mathrm{Feb} / 2009 & 9 \mathrm{ss} / 3 / 3 & 9 \mathrm{ss} / 1 / 3 & 9 \mathrm{ss} / 2 / 3 & 9 \mathrm{ss} / 2 / 2 & 9 \mathrm{ss} / 4 / 2 & 9 \mathrm{ss} / 4 / 3\end{array}$

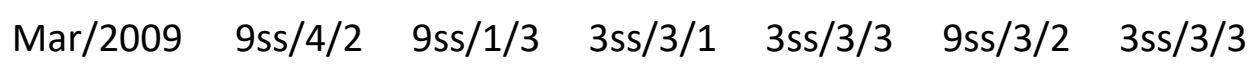

Mar/2009 $3 s s / 4 / 2 \quad 3 s s / 2 / 3 \quad 3 s s / 4 / 2 \quad 3 s s / 1 / 1 \quad 3 s s / 2 / 1 \quad 3 s s / 2 / 3$

Apr/2009 3ss/2/1 $3 s s / 1 / 2 \quad 3 s s / 1 / 2 \quad 9 s s / 1 / 1 \quad 9 s s / 4 / 1 \quad 3 s s / 4 / 3$

$\begin{array}{lllllll}A p r / 2009 & 9 s s / 3 / 3 & 9 s s / 3 / 2 & 3 s s / 2 / 2 & 3 s s / 3 / 2 & 9 s s / 3 / 3 & 3 s s / 4 / 3\end{array}$

$\begin{array}{lllllll}\text { May/2009 } & 3 s s / 4 / 1 & 3 s s / 4 / 1 & 9 s s / 2 / 1 & 9 s s / 2 / 2 & 9 s s / 2 / 3 & 3 s s / 1 / 3\end{array}$

560 Table A1. 30 training tanks were set up, fish were trained in blocks of 5 (15 replicates per

561 species per treatment=5 blocks). See main text for further details. Codes indicate species /

562 training / testing, where: Species: 3ss / 9ss = three-spined sticklebacks / nine-spined sticklebacks.

563 Training: 1/2/3/4: 1 . Test subjects and demonstrators both fed from floating tile / 2 . Test subjects

564 fed from tile, demonstrators fed from substrate / 3. Test subjects fed from substrate,

565 demonstrators fed from tile / 4. Test subjects and demonstrators both from substrate. Testing: 
566 1/2/3: 1. Demonstrators present and feeding. 2. Demonstrators present but not feeding. 3. No

567 demonstrators present.

568

569

570

571

572

573

574

575

576

577

578

579

580

581

582

583

584

585

586

587

588 
591 Figure 1. Training and testing regimes. Fish were trained in one of four treatments: Training: 1.

592 Test subjects and demonstrators both fed from floating tile (grey block in figure). 2. Test subjects

593 fed from tile, demonstrators fed from substrate. 3. Test subjects fed from substrate,

594 demonstrators fed from tile. 4. Test subjects and demonstrators both from substrate. Fish from

595 each training regime were then test in one of three treatments: Testing: 1 . Demonstrators present

596 and feeding. 2. Demonstrators present but not feeding. 3. No demonstrators present.

598 Figure 2. The height above the base of the tank in $\mathrm{cm}$ (mean $+/-95 \% \mathrm{CI}, N=15$ trials per data 599 point) seen in test fish trained under different training regimes (legend) and tested under one of 600 three treatment conditions (P, F: conspecifics present and feeding; P, NF: present but not 601 feeding; A: conspecifics absent). Rising higher above the base of the tank is interpreted as food 602 anticipatory behaviour.

604 Figure A1. The height above the base of the tank in $\mathrm{cm}$ (mean $+/-95 \% \mathrm{CI}, N=15$ trials per data 605 point) seen in three-spined sticklebacks trained under the four different training regimes and 606 tested under one of three treatment conditions [(a) Demonstrators present and feeding, (b) 607 Demonstrators present but not feeding, (c) No demonstrators present]. Rising higher above the 608 base of the tank is interpreted as food anticipatory behaviour. Data show mean height per minute 609 during the five minute duration tests. Since we saw no major trends over the trial duration we 610 used whole trial means in the analysis presented in the main text and for the data presented in 611 Figure 2. 
612 Figure A2. The height above the base of the tank in $\mathrm{cm}$ (mean $+/-95 \% \mathrm{CI}, N=15$ trials per data

613 point) seen in nine-spined sticklebacks trained under the four different training regimes and

614 tested under one of three treatment conditions [(a) Demonstrators present and feeding, (b)

615 Demonstrators present but not feeding, (c) No demonstrators present]. Rising higher above the

616 base of the tank is interpreted as food anticipatory behaviour. Data show mean height per minute

617 during the five minute duration tests. Since we saw no major trends over the trial duration we

618 used whole trial means in the analysis presented in the main text and for the data presented in

619 Figure 2.

620

621

622

623

624

625

626

627

628

629

630

631

632

633

634 
635

636

Training

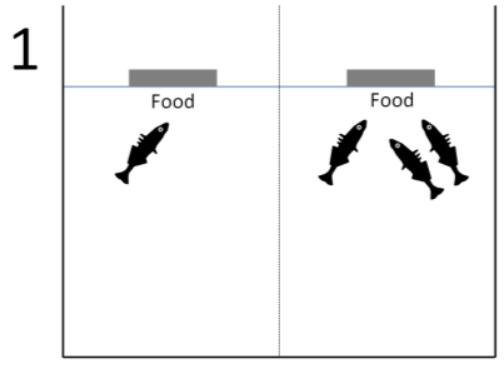

2

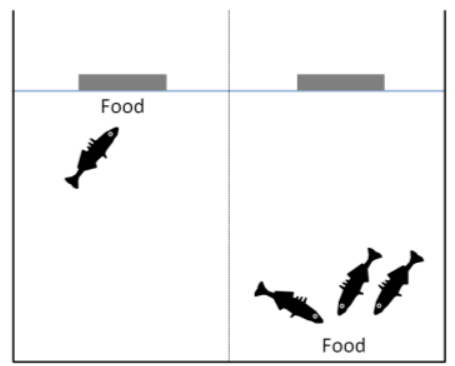

3

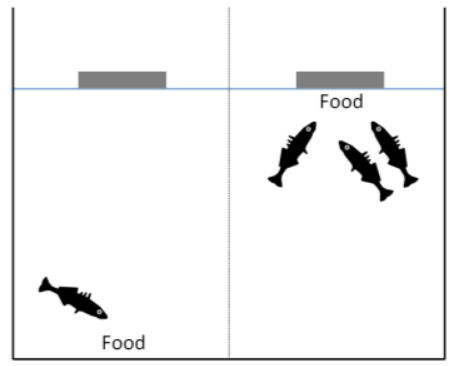

4

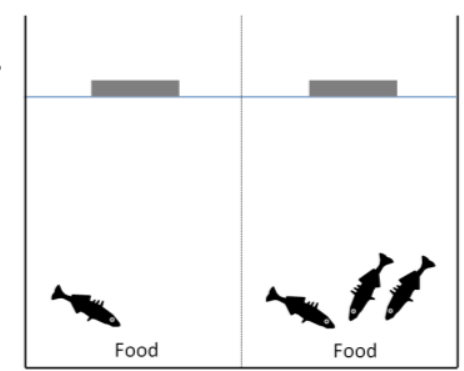

637

Testing
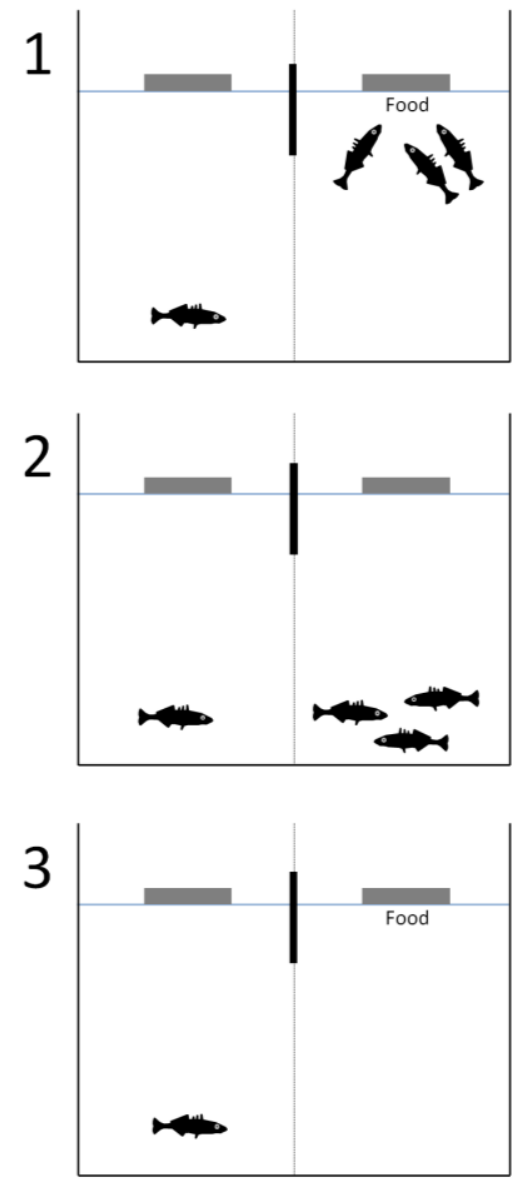

638

639

640

641

642 
Figure 2.

644

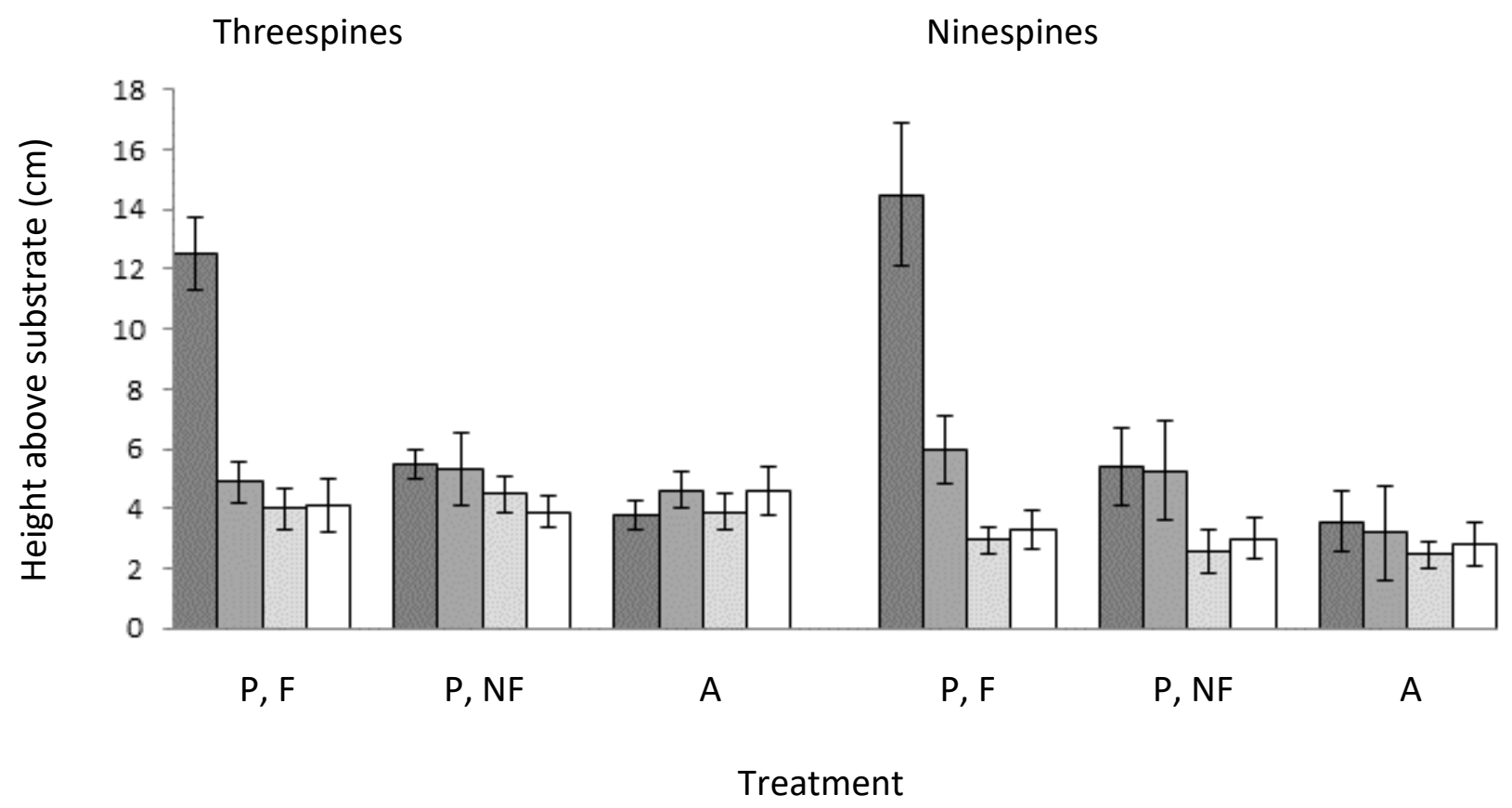

645

646 Experience of feeding from floating tile and seeing others eat from it

647 Experience of feeding from floating tile but not of seeing others eat from it

$648 \quad$ No experience of feeding from tile, but has seen others feed from it

$649 \square$ No experience of feeding from tile or seeing others feed from it

650

651

652

653

654

655

656

657

658

659 
660

661

\section{Figure A1.}
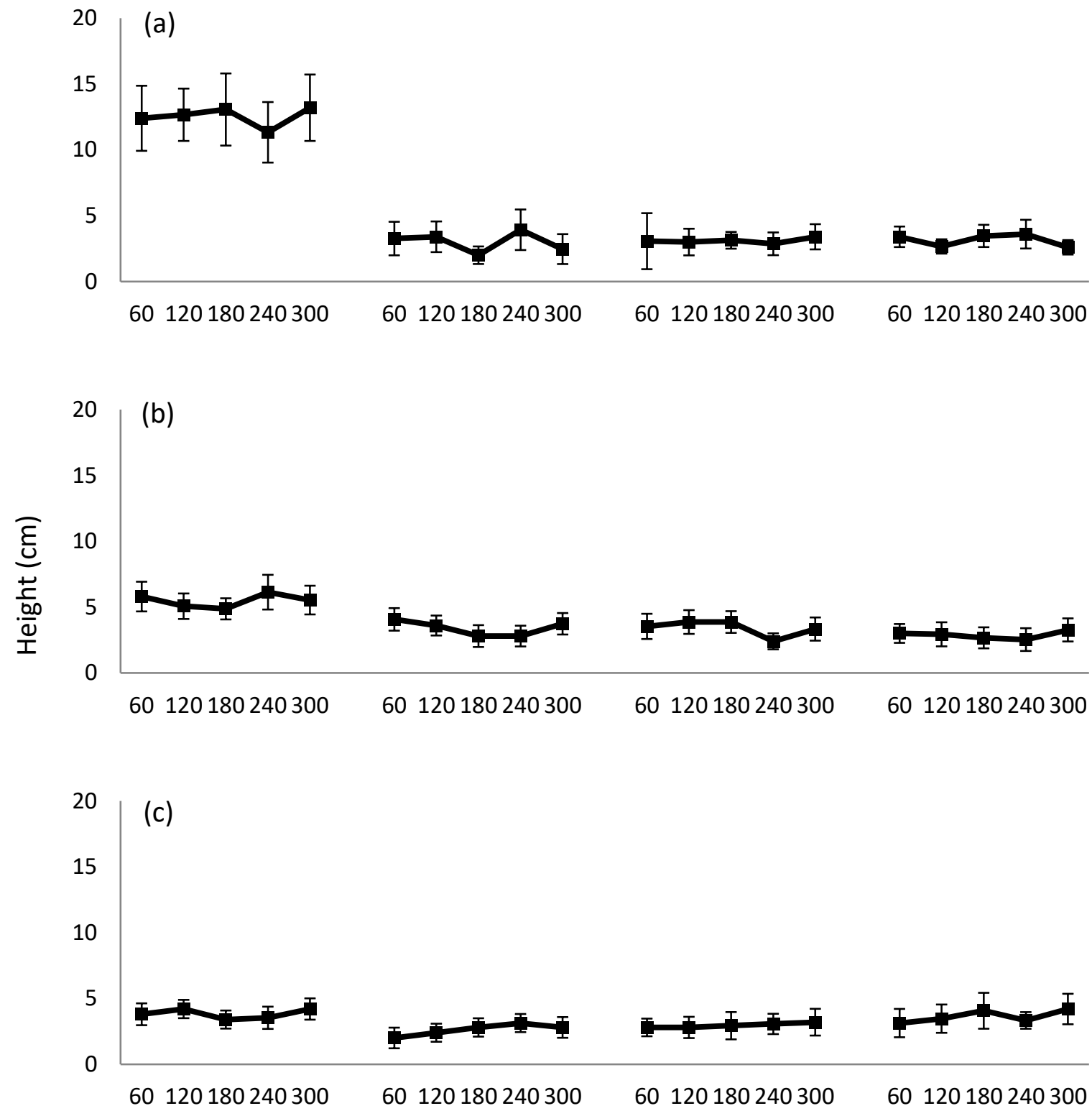

662

663

664

665
Time (s)
Training 1
Training 2
Training 3
Training 4 
Figure A2.
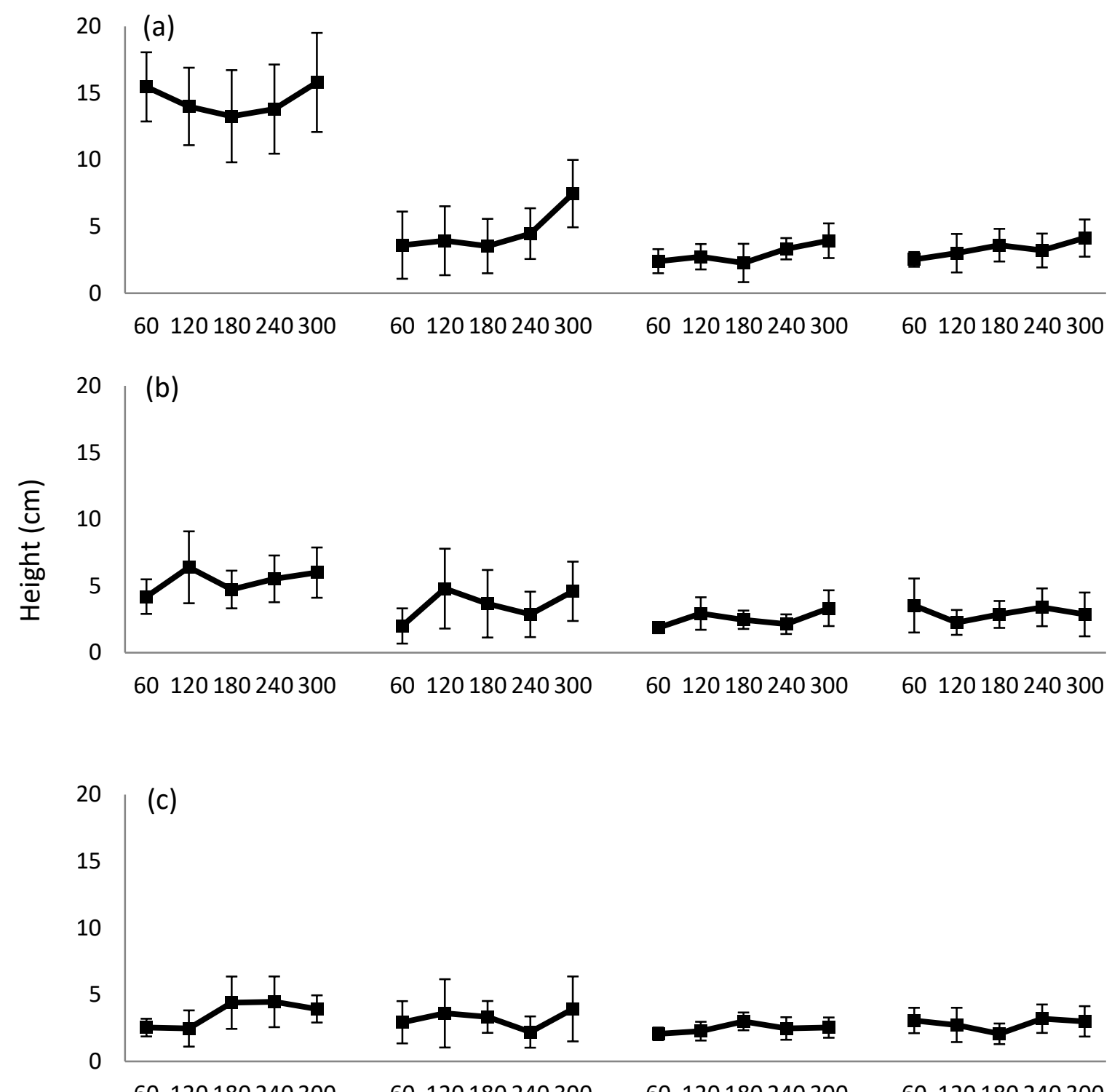